\title{
Mechanism of Dissolution Enhancement and Bioavailability of Poorly Water Soluble Celecoxib by Preparing Stable Amorphous Nanoparticles
}

\author{
Yinghui Liu ${ }^{\mathrm{a}}$, Changshan Sun ${ }^{\mathrm{a}}$, Yanru Hao ${ }^{\mathrm{b}, \mathrm{a}}$, Tongying Jiang ${ }^{\mathrm{a}}$, Li Zheng ${ }^{\mathrm{a}}$, Siling Wang ${ }^{\mathrm{a}}$ \\ a Department of Pharmaceutics, School of Pharmacy, Shenyang Pharmaceutical University, No.103, Wenhua Road, \\ Shenyang 110016, PR China. \\ bShenyang B. Braun Pharmaceutical Co., Ltd, Shenyang 110003, PR China.
}

Received, September 24, 2010; Revised, November 29, 2010; Accepted December 7, 2010; Published December 9, 2010.

\begin{abstract}
Purpose: Nanoparticle engineering offers promising methods for the formulation of poorly water soluble drug compounds. The aim of the present work was to enhance dissolution and oral bioavailability of poorly water-soluble celecoxib (CXB) by preparing stable CXB nanoparticles using a promising method, meanwhile, investigate the mechanism of increasing dissolution of CXB. Methods: CXB nanoparticles were produced by combining the antisolvent precipitation and high pressure homogenization $(\mathrm{HPH})$ approaches in the presence of HPMC E5 and SDS $(2: 1, \mathrm{w} / \mathrm{w})$. Then the CXB nanosuspensions were converted into dry powders by spray-drying. The effect of process variables on particle size and physical state of CXB were investigated. The physicochemical properties of raw CXB and CXB nanoparticles were characterized by scanning electron microscopy (SEM), X-ray powder diffraction (XRPD), X-ray photoelectron spectra (XPS), fourier transform infrared (FT-IR) spectroscopy, diffrential scanning calorimetry (DSC), as well as, measuring the particle size and contact angle. Additionally, the studies of in-vitro drug dissolution and oral bioavailability in beagle dogs of nanoparticles were performed. Results: The images of SEM revealed spherical CXB nanoparticles. The DSC and XRPD results indicated that the antisolvent precipitation process led to the amorphization of CXB. Under storage, the amorphous CXB nanoparticles showed promising physical stability. The XPS data indicated the amorphous CXB nanoparticles exhibited different surface property compared to raw CXB. Hydrogen bonds were formed between the raw CXB and HPMC E5 as proven by the FT-IR spectra. CXB nanoparticles increased the saturation solubility of CXB fourfold. CXB nanoparticles completely dissolved in the dissolution medium of phosphate buffer ( $\mathrm{pH} 6.8,0.5 \% \mathrm{SDS}$ ) within $5 \mathrm{~min}$, while there was only $30 \%$ of raw CXB dissolved. The $\mathrm{C}_{\max }$ and $\mathrm{AUC}_{0-24 \mathrm{~h}}$ of $\mathrm{CXB}$ nanoparticles were approximately threefold and twofold greater than those of the Celecoxib Capsules, respectively. Conclusions: The process by combining the antisolvent precipitation under sonication and HPH was a promising method to produce small, uniform and stable CXB nanoparticles with markedly enhanced dissolution rate and oral bioavailability due to an increased solubility that is attributed to a combination of amorphization and nanonization with increased surface area, improved wettability and reduced diffusion pathway.
\end{abstract}

\section{INTRODUCTION}

The poor solubility and slow dissolution rate of many drugs are a major industrial problem, especially for pharmaceutical scientists involved in drug discovery and drug development. It has been reported that about $40 \%$ of the compounds being developed by the pharmaceutical industry are poorly water soluble or "insoluble" in water (1-2). Poor water solubility is the major hurdle to be overcome in the case of poorly water soluble drugs in terms of their vivo performance due to their inadequate ability to be wetted by and dissolved in the fluid in the gastrointestinal tract after oral administration; this is because dissolution is the first step in the absorption of the drugs (2). Hence, improving the saturation solubility and dissolution rate of poorly water soluble drugs is very important and significantly challenging to pharmaceutical researchers seeking to achieve optimum absorption of new drug candidates. According to the Noyes-Whitney equation, the saturation solubility and dissolution rate of poorly water soluble drugs can be enhanced by reducing the particle size to the nano-range, thus increasing the interfacial surface area (3-4).

Corresponding Author: Dr. Siling Wang, Department of Pharmaceutics, School of Pharmacy, Shenyang Pharmaceutical University, No.103, Wenhua Road, Shenyang 110016, PR China; Email: silingwang@syphu.edu.cn 
In recent years, nanoparticle engineering processes have become promising approaches for the enhancement of dissolution rates of poorly aqueous soluble drugs (5-7). At present, the preparation of drug nanoparticles can be basically classified into two categories: (a) the top-down approach and (b) the bottom-up approach. Top-down process is a common, scalable and effective mechanical process used to produce nanometer-sized particles of poorly aqueous soluble drug compounds. Nowadays, there have been five marketed products using this approach (Table 1). The formation of nanoparticles is based on cavitation forces, collisions as well as shear forces created in high pressure homogenizers such as the piston-gap homogenizer (8). The bottom-up process, antisolvent precipitation under sonication is a relatively rapid and low cost method. In this method, the drug is dissolved in an organic solvent and then added to an antisolvent in the presence of surfactants, polymers or a mixture of both as stabilizers. The key to preparing nanoparticles by antisolvent precipitation is to create conditions that favor very rapid particle formation and little or no particle size growth. Ultrasound has been used to generate nano-sized drug particles by (a) intensifying the mass transfer avoiding inefficient mixing; (b) atomizing the drug solution with very fine droplets into the antisolvent resulting in the formation of smaller particles; (c) reducing the particle size of the newly formed particles and suppressing the agglomeration of fine particles (9-12).

Currently, nano-sized particles of some poorly water soluble drugs have been produced by a top-down or bottom-up approach alone, however, researchers have rarely obtained nanoparticles by a combination of the bottom-up and top-down approaches. To our knowledge no study regarding the CXB nanoparticles prepared by antisolvent precipitation under sonication has been yet published. Although it was not very difficult to prepare nanoparticles of many poorly water soluble drugs, the stability of drug nanoparticles was very crucial to ensure the advantages of nano-sized particles. Therefore it is imperative to produce $\mathrm{CXB}$ nanoparticles having the long term stability under storage. Celecoxib (Figure 1) is the first selective cyclo-oxygenase-2 (COX-2) enzyme inhibitor introduced to the anti-inflammatory drug market. It belongs to the BCS class II substance (13-16). CXB is weakly acidic and has a low solubility which is low at $3-7 \mu \mathrm{g} / \mathrm{mL}$ when determined in vitro at $\mathrm{pH} 7.0$ and $40{ }^{\circ} \mathrm{C}$ (17). It has been reported that the bioavailability of the conventional capsule dosage form ranges from 22 to $40 \%$ in dogs and the extent of drug absorption is limited by the dissolution rate (18-19). Low solubility is expected to contribute to the highly variable absorption after oral administration. Hence, increasing the solubility of CXB may enhance its oral bioavailability. Although many researchers have been attempted to improve the solubility by various methods such as complexation with beta-cyclodextrin (20), self-emulsifying (21) and microemulsions (22-23), conclusive evidence for the formulation of CXB in dosage forms with adequate solubility and bioavailability are rare. Dolenc Andrej et al. (24) have produced CXB nanosuspensions existing as crystalline form by the emulsion-diffusion (solvent exchange) method but with a broad particle size distribution (PSD) of $0.14-1.29 \mu \mathrm{m}$. They found the nano-sized to readily assemble and grow resulting in great variation of the dissolution rate. Ram $R$. et al. (25) have encapsulated in the nanostructured lipid carrier in order to prepare nanostructured lipid carrier nanoparticles of CXB for pulmonary delivery, obtaining improved CXB pulmonary bioavailability. The results indicated that nano-CXB is promising. Katrin Margulis-Goshen et al. (26) have obtained CXB nanopowder by rapid evaporation of drug-loaded volatile microemusions achieving high enhanced dissolution compared to the particulate drug in the presence of surfactants. However, relatively high amount of excipients in the microemulsions may increase the side effects and toxicity of the formulation.

Drugs would exhibit some distinct properties when they were reduced to nano-range (27). However, the investigation on the properties of CXB nanoparticles that favors dissolution and bioavailability was limited.

The aim of this study was to prepare and investigate the properties and mechanisim of enhancement of dissolution and oral bioavailability of the stable CXB nanoparticles. Although CXB nanoparticles can be prepared by antisolvent precipitation under sonication, the PSD of CXB particles was broad. Thus, we made an attempt on using antisolvent precipitation followed by HPH. The physicochemical properties of $\mathrm{CXB}$ nanoparticles were investigated. In addition, improvement on in-vitro drug dissolution and oral bioavailability in beagle dogs of nanoparticles was validated. 


\begin{tabular}{llll}
\hline Table 1. Available commercial products relying on drug nanoparticle technology & \\
\hline Product and active ingredient & Manufacturing approach & Date of FDA approval & Company \\
\hline Rapamune ${ }^{\circledR}$, Sirolimus & Top-down, media milling & August 2000 & Wyeth \\
Emend ${ }^{\circledR}$, Aprepitant & Top-down, media milling & March 2003 & Merck \\
TriCor ${ }^{\circledR}$, Fenofibrate & Top-down, media milling & November 2004 & Abbott \\
Megace ${ }^{\circledR}$ ES, Megestrol acetate & Top-down, media milling & July 2005 & Par Pharmaceutica \\
Triglide ${ }^{\circledR}$, Fenofibrate & Top-down, HPH & May 2005 & Skye Pharma \\
\hline
\end{tabular}

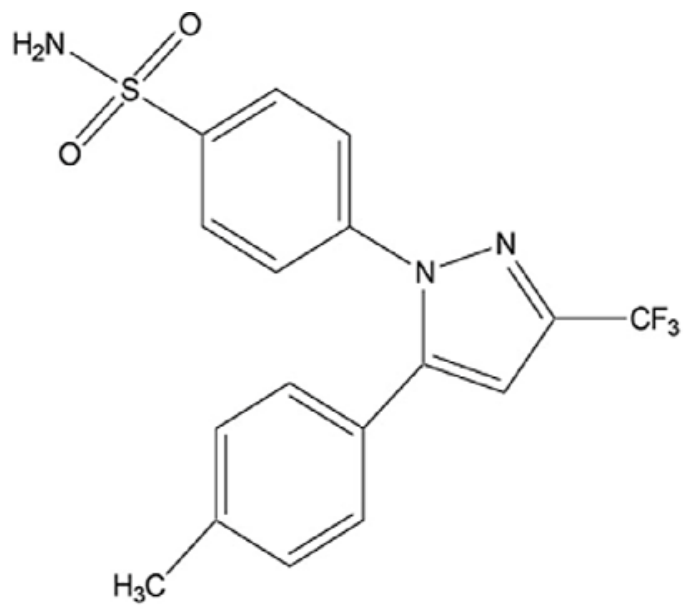

Figure 1. Chemical structure of $\mathrm{CXB}$

\section{MATERIALS AND METHODS}

\section{Materials}

Celecoxib USP30 was purchased from Guangzhou Eastbang Pharmaceutical Co., Ltd (China). Hydroxypropyl methylcellulose (HPMC E5) was a generous gift from Dow Chemical Company (America). Pluronic F-68, Pluronic F-127 and PVP K-30 were kindly gifted by BASF (D-Ludwigshafen, Germany). Sodium dodecyl sulfate (SDS) and Tween 80 were obtained from Tianjin Bodi Chemical Holding Co., Ltd (China). Methanol and ethyl acetate was of chromatographic grade and all other chemicals, reagents and solutions used were of analytical grade.

\section{Preparation of CXB nanosuspensions}

CXB nanosuspensions were prepared in the following two steps. Firstly, the initial CXB suspensions were produced by antisolvent precipitation under sonication. Acetone and water were used as solvent and antisolvent respectively and the ratio of solvent to antisolvent was 1:20.
Briefly, $600 \mathrm{mg}$ raw CXB was completely dissolved in $10 \mathrm{~mL}$ acetone to prepare an organic solution of $\mathrm{CXB}$, and this solution was then injected into $200 \mathrm{~mL} 0.15 \%(\mathrm{w} / \mathrm{v})$ aqueous solution (containing HPMC E5 and SDS, 2:1, $\mathrm{w} / \mathrm{w}$ ) maintained at $4{ }^{\circ} \mathrm{C}$ under sonication at 300 $\mathrm{W}$ within $3 \mathrm{~min}$. Continuous sonication was applied via a probe sonicator (JY92-II, Scientz, Ningbo, China) for intermittent sonication lasting $3 \mathrm{~s}$ at $2 \mathrm{~s}$ intervals for a total sonication time of 3 min. Thus, preliminary $\mathrm{CXB}$ suspensions were obtained and labeled as SO-CXB. The suspensions were kept under vacuum at room temperature for $2 \mathrm{~h}$ to remove the acetone. Then, the CXB suspensions were further homogenized by $\mathrm{HPH}$, using an ATS AH100D homogenizer (ATS Engineer Inc. China) at 500 bar for 8 min to obtain the final product, CXB nanosuspensions labeled as HSO-CXB. The temperature of the suspensions was maintained at $-10{ }^{\circ} \mathrm{C}$ during the process using a circulating ethanol bath (DL-1005, Ningbo Scientz Biotechnology Co., Ltd, China). 


\section{Spray-drying of CXB nanosuspensions}

Spray-drying was used for solidification of CXB nanosuspensions instead of lyophilisation. Spray-drying is generally employed by the pharmaceutical industry convert nanosuspensions to dry products because it requires less time and energy. A spray dryer (SD-1000, Tokyo Rikakikal. Co., Ltd., Japan) was used to convert CXB nanosuspensions into dry powders. The inlet air temperature was $105{ }^{\circ} \mathrm{C}$. The aspirator was operated at $0.6 \mathrm{~m}^{3} / \mathrm{min}$ and the pump was set at 5 $\mathrm{mL} / \mathrm{min}$. $200 \mathrm{~mL}$ CXB nanosuspensions was not diluted before spray-drying and no protectant was added. The dry powder with nano-sized CXB exhibited good uniformity and the yield of dry powder was approximately $80 \%$.

\section{Characterization of CXB nanoparticles Particle size analysis}

Particle size analysis of the CXB nanosuspensions was performed by Laser diffraction using a Coulter LS 230 instrument from Beckmann Coulter Electronics (Krefeld, America). Laser diffraction measures volume-weighted particle size distribution over the size range 0.040 to 2000 $\mu \mathrm{m}$ particle-size distribution which typically includes $d(10), d(50)$ and $d(90)$ representing the percentage of particles below a given size $(\mu \mathrm{m})$. The nanoparticle suspensions were analyzed without dilution.

\section{$X$-ray photoelectron spectroscopy}

$\mathrm{X}$-ray photoelectron spectroscopy, also known as electron spectroscopy for chemical analysis (ESCA), was used to probe the elemental composition of the powder surfaces with an analysis depth of less than $100 \AA$. X-ray photoelectron spectra were recorded for powder sample using a high vacuum X-ray photoelectron spectroscope (ESCALAB250, Thermo VG, USA) with $\mathrm{Al} \mathrm{K}_{\alpha} \mathrm{X}$-ray source of $150 \mathrm{~W}$ with operating voltage of $15 \mathrm{kV}$. Binding energy range was from 0 to $1300 \mathrm{eV}$ and the average peak binding energy of $\mathrm{C} 1 \mathrm{~s}, \mathrm{~N} 1 \mathrm{~s}, \mathrm{O} 1 \mathrm{~s}, \mathrm{~F} 1 \mathrm{~s}$ and $\mathrm{S} 2 \mathrm{p}$ were 285 , $399.81, \quad 532.19, \quad 688.19$ and $168.72 \mathrm{eV}$, respectively. All spectra were baseline subtracted and fitted using Gaussian function. The data for the atomic surface composition were converted into molecular surface composition under certain assumptions. Prior to solidification the excess of HPMC E5 and SDS was removed by ultracentrifugation at $15,000 \mathrm{r} / \mathrm{m}$ for $30 \mathrm{~min}$.

\section{Scanning electron microscopy}

The morphologies of raw CXB and nano-sized CXB were examined using a scanning electron microscope (JEOL JSM-7001F, Japan) operated at an accelerating voltage of $15 \mathrm{kV}$ and a secondary detector. Freshly prepared CXB nanosuspensions and dispersions of raw $\mathrm{CXB}$ were deposited on a glass slides following the evaporation of solvent and coated with gold.

\section{Differential scanning calorimetry}

A differential scanning calorimeter DSC-60 (Shimadzu, Japan) was calibrated using an indium standard. The samples were placed in hermetically sealed aluminum pans and heated from 30 to $200{ }^{\circ} \mathrm{C}$ at $10{ }^{\circ} \mathrm{C} / \mathrm{min}$ and with a nitrogen purge of $40 \mathrm{~mL} / \mathrm{min}$.

\section{$X$-ray powder diffraction}

X-ray powder diffraction measurements were carried out on samples using a diffractometer (Rigaku Geigerflex XRD, Co., Japan) with $\mathrm{Cu}-\mathrm{Ka}$ radiation $(\lambda=1.54 \AA)$ at $30 \mathrm{kV}$ and $30 \mathrm{~mA}$. Data were collected from 5 to $40^{\circ} 2 \theta$ with 0.02 steps.

\section{Fourier transform infrared spectroscopy}

The FT-IR spectra of samples were obtained, after appropriate background subtraction using an FT-IR spectrometer (Bruker IFS 55, Switzerland). Samples were diluted with dry potassium bromide and scanned from $4000-400 \mathrm{~cm}^{-1}$ to evaluate the molecular states of raw $\mathrm{CXB}$ and nano-sized CXB.

\section{Contact angle}

The contact angle measurements were employed to describe the wettability of the spray-dried powder with nano-sized CXB compared with raw CXB. Pure CXB powder and spray-dried powder with nano-sized $\mathrm{CXB}$ were compressed into tablets (200 mg powder, $5 \pm 0.4 \mathrm{~kg}$ of pressure) to determine their contact angles. The contact angle measurements on tablets were performed using a Dynamic Absorption Tester (JY-82, Chengde Dingsheng Tester Equipment Co., Ltd, China) and water was dripped on to the tablets followed by image capture (a CCD camera) and analysis, to obtain parameters such as the drop volume, base diameter and contact angle. Three measurements per sample were performed to ensure reproducibility.

\section{Determination of saturation solubility and in vitro drug release}

Apparent saturation solubility measurements were carried out on raw $\mathrm{CXB}$, a physical mixture and spray-dried powder with nano-sized CXB. A known excess of sample was added to $10 \mathrm{~mL}$ double-distilled water $\left(\mathrm{pH} 7.00\right.$ at $\left.20{ }^{\circ} \mathrm{C}\right)$ which was then stirred at $100 \mathrm{r} / \mathrm{m}$ in an air bath $\left(37 \pm 0.3{ }^{\circ} \mathrm{C}\right)$ for $24 \mathrm{~h}$. The double-distilled water 
was obtained by using an automatic double pure water distiller (SZ-93A, Shanghai Yarong Biochemical Instrument Factory, China). After centrifugation and filtration through $0.22 \mu \mathrm{m}$ filter paper, concentration of CXB was determined spectrophotometrically at $253 \mathrm{~nm}$. Every sample was analyzed in triplicate and the mean values and standard deviations were reported.

A dissolution study was carried out following the USP Apparatus 2 (paddle) method (RC-8D, Tianjin Guoming Medical Equipment Co., Ltd, China). The paddle speed and bath temperature were set at $100 \mathrm{r} / \mathrm{m}$ and $37.0 \pm 0.5^{\circ} \mathrm{C}$, respectively. Phosphate buffer ( $\mathrm{pH} 6.8,0.5 \%$ SDS) was used as the dissolution medium. Approximately $50 \mathrm{mg}$ powder was added to vessels containing $900 \mathrm{~mL}$ of dissolution medium. Samples $(4 \mathrm{~mL})$ were collected periodically and replaced with a fresh dissolution medium. After centrifugation and filtration through $0.22 \mu \mathrm{m}$ filter paper, concentration of CXB was determined spectrophotometrically at $253 \mathrm{~nm}$ after suitable dilution.

\section{Bioavailability study Experimental procedure}

Male and female pure-bred beagle dogs weighing between $12-15 \mathrm{~kg}$ were used. Dogs $(\mathrm{n}=6)$ were fasted overnight before dosing and were given access to food approximately $6 \mathrm{~h}$ postdose. Dogs were administered CXB orally in a Celecoxib Capsules (Pfizer Pharmaceuticals LLC) as control and suspension of spray-dried powder (prepared in water just before dosing) nanoparticles in a randomized, crossover design at a dose of 5 $\mathrm{mg} / \mathrm{kg}$ (18). All animal experiments were performed according to the "Guidelines for the Care and Use of Laboratory Animals" at Shenyang Pharmaceutical University. The protocol was approved by our university's Animal Ethics Committee. Venous blood (approximately $4 \mathrm{~mL}$ ) from the jugular vein was collected into chilled tubes containing sodium heparin from the animals at approximately $0.5,0.75,1,1.5,2,2.5$, $3,3.54,6,812$ and $24 \mathrm{~h}$ after the oral doses. Plasma was separated by centrifugation at 3000 $\mathrm{r} / \mathrm{m}$ for $10 \mathrm{~min}$ and then stored at $-20{ }^{\circ} \mathrm{C}$ for analyzing by HPLC.

\section{Assay of plasma concentration}

Frozen plasma samples were thawed and allowed to reach room temperature. A $500 \mu \mathrm{L}$ aliquot of plasma was placed into a test tube, $50 \mu \mathrm{L}$ internal standard solution $(5 \mu \mathrm{g} / \mathrm{mL})$ and $2 \mathrm{~mL}$ ethyl acetate were added, respectively. The tubes were mixed by vortexing for $5 \mathrm{~min}$, centrifuged at $12000 \mathrm{r} / \mathrm{m}$ for $5 \mathrm{~min}$. The organic layer was transferred to a clean test tube and evaporated by centrifugal at $40{ }^{\circ} \mathrm{C}$ (Labconco Centrivap, USA). The residues were reconstituted in $100 \mu \mathrm{L}$ of mobile phase, mixed well and $20 \mu \mathrm{L}$ of the final clear solution was injected into the HPLC system. Separation was achieved using a HPLC system Hitachi L-7000 series consisting of a pump (Hitachi L7100) and a UV detector (Hitachi L-7400) (Tokyo, Japan) equipped with a Kromasil C18 column $(250 \mathrm{~mm} \times 4.6 \mathrm{~mm} 5 \mu \mathrm{m}$, Sweden $)$. The isocratic mobile phase consisted of methanol:water (69:31, v/v) prepared daily and degassed by passing through a $0.45 \mu \mathrm{m}$ filter. All chromatographic separations were performed at the flow rate of $0.9 \mathrm{~mL} / \mathrm{min}$ at room temperature. The UV detection was performed at $253 \mathrm{~nm}$. Carbamazepine was used as internal standard.

Calibration curves were constructed by plotting the measured peak area ratios of CXB to Carbamazepine versus concentrations of standard samples. The \% recovery of CXB from plasma was estimated from $\%$ recovery $=(\mathrm{CXB}$ peak area in plasma sample /CXB peak area in methanol sample) $\times 100$. The percent recovery of CXB from plasma in the lowest, medium and highest concentration were determined to be $106 \pm 7.9$, $87.8 \pm 9.3,86.7 \pm 5.6$ and $90.3 \pm 3.7 \%$ for 10,100 , 400 and $1000 \mathrm{ng} / \mathrm{mL}$, respectively. For carbamazepine, The \% recovery from plasma was $79.8 \pm 9.9 \%$. The short term stability of the samples during analysis was tested in the lowest, medium and highest concentration at $0,2,4,8,12$ and $24 \mathrm{~h}$ after preparation. We set the lowest limit of quantification at $10 \mathrm{ng} / \mathrm{mL}$. The analysis of all data was performed using DAS 2.1.1 software. The assay validation data are presented in Table 2.

\section{RESULTS}

\section{Preparation of CXB nanoparticles and particle} size analysis

In this work, CXB nanoparticles were successfully prepared by the combination of antisolvent precipitation and HPH. The PSD of particles prepared by the different approaches was represented in Table 3. It can be clearly seen that the PSD of particles prepared only by antisolvent precipitation under sonication was not narrow enough and, hence, HPH was employed followed by antisolvent precipitation under sonication. Consequently, the particle size of large particles decreased dramatically with a narrower PSD, and the mean size was $159 \pm 15 \mathrm{~nm}$ (Table 3, Figure 2 500 bar). 
Table 2. Precision and accuracy of CXB assay ( $\mathrm{n}=15$; five sets for 3 days)

\begin{tabular}{|c|c|c|c|}
\hline \multicolumn{2}{|c|}{ Concentration, $\mathrm{ng} / \mathrm{mL}$} & \multirow[t]{2}{*}{$\mathrm{CV}$} & \multirow{2}{*}{$\frac{\text { Accuracy }}{\text { Percent }}$} \\
\hline Added & Observed & & \\
\hline \multicolumn{4}{|c|}{ Intra-day $(\mathrm{n}=5)$, Mean \pm S.D. } \\
\hline 10 & $9.94 \pm 0.36$ & 3.62 & -0.6 \\
\hline 100 & $98.94 \pm 0.86$ & 0.87 & -1.06 \\
\hline 400 & $403.36 \pm 1.68$ & 0.42 & 0.84 \\
\hline 1000 & $1001.45 \pm 2.78$ & 0.28 & 0.15 \\
\hline \multicolumn{4}{|c|}{ Inter-day $(n=15)$, Mean \pm S.D. } \\
\hline 10 & $10.26 \pm 0.28$ & 2.73 & 2.6 \\
\hline 100 & $98.87 \pm 1.26$ & 1.27 & -1.13 \\
\hline 400 & $403.76 \pm 2.78$ & 0.69 & 0.94 \\
\hline 1000 & $1002.65 \pm 3.87$ & 0.39 & 0.27 \\
\hline
\end{tabular}

Table 3. The volume-weighted PSD of CXB micro/nanoparticles obtained by different processes: SO-CXB, prepared only by antisolvent precipitation under sonication; HSO-CXB, prepared by combining the antisolvent precipitation under sonication and HPH (500 bar, 15cycles). (CXB acetone concentration: $60 \mathrm{mg} / \mathrm{mL}$; stabilizer concentration: $0.15 \%, \mathrm{w} / \mathrm{v}), \mathrm{n}=3$

\begin{tabular}{lcccc}
\hline Particles size $(\mu \mathrm{m})$ & $\mathrm{d}(10)$ & $\mathrm{d}(50)$ & $\mathrm{d}(90)$ & Mean \pm S.D. \\
\hline SO-CXB & 0.111 & 0.259 & 8.111 & $2.568 \pm 0.022$ \\
HSO-CXB & 0.0988 & 0.151 & 0.231 & $0.159 \pm 0.015$ \\
HSO-CXB $^{\mathrm{a}}$ & 0.143 & 0.165 & 0.290 & $0.170 \pm 0.018$ \\
HSO-CXB $^{\mathrm{b}}$ & 0.153 & 0.194 & 0.311 & $0.211 \pm 0.019$ \\
\hline
\end{tabular}

Note: ${ }^{a}$ particle size of HSO-CXB after spray drying; ${ }^{b}$ particle size of HSO-CXB after two months.

\section{Effects of stabilizer and stabilizer concentration}

It is well known that an appropriate stabilizer is very important to control particle growth during the production of uniform nanoparticles. Therefore, various surfactants and polymers, such as SDS, HPMC, PVP K30, PVA, F-68, F-127 and Tween 80 (used alone or a mixture of both) were screened by trials in terms of their particle sizes, PSD and nanosuspensions stability. When a combination of HPMC E5 and SDS $(2: 1, w / w)$ was selected as the stabilizer, smaller submicron particles with a narrow PSD were obtained in comparison with the other stabilizers cited above (Figures not shown). Furthermore, the effect of the stabilizer concentration on the particle size ranging from $0.03,0.06,0.1,0.15,0.3,0.6$ to 0.9 $(\%, \mathrm{w} / \mathrm{v})$ was investigated. When the stabilizer concentration was below $0.1 \%$, the mean particle size was about $800 \mathrm{~nm}$ and can be increased easily. As the stabilizer concentration increased to $0.15 \%$, the mean particle size was dramatically reduced to $159 \mathrm{~nm}$ and particle growth can be controlled well by stabilizers. Following a further increase in stabilizer concentration, the particle size was not markedly reduced, which indicated that the drug particle surface was already sufficiently enveloped by the stabilizer molecules.

\section{Effects of homogenization pressure and number of homogenization cycles}

The pressure and number of cycles are two important parameters when producing nanoparticles by HPH. Final CXB nanoparticles were obtained by antisolvent precipitation under sonication followed by HPH. The particle size of CXB nanoparticles at different pressures was showed in Figure 2. The particle size of large particles was reduced as the pressure increased from 300 to 500 bar ( 15 cycles), but the particle size was not markedly reduced at a higher homogenization pressure (700-900 bar). The number of cycles had a greater influence on PSD in comparison with the particle size when the pressure was constant. A further increase in the number of cycles had the same effect as the pressure increased. 


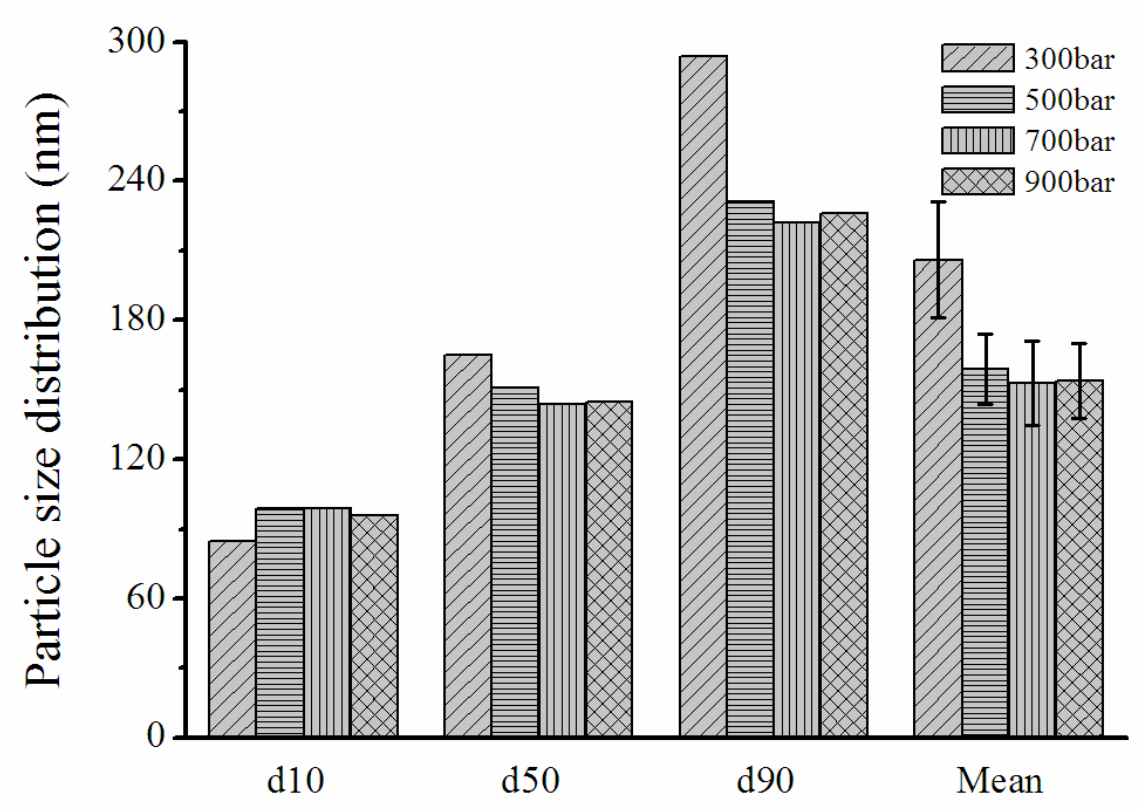

Figure 2. Volume-weighted PSD of CXB nanoparticles prepared under different homogenization pressures (CXB concentration: $60 \mathrm{mg} / \mathrm{ml}$; stabilizer concentration: $0.15 \%$, w/v; acetone/water volume ratio: $1 / 20 ; 15$ cycles), $\mathrm{n}=3$

\section{The morphology of CXB nanoparticles}

The SEM images are showed in Figure 3. It can be seen that the raw drug particles existed as predominantly needle-shaped crystals (Figure 3, a). However, CXB nanoparticles obtained by the combination of antisolvent precipitation and HPH were spherical with a narrow PSD in the presence of a combination of HPMC and SDS $(2: 1, \mathrm{w} / \mathrm{w})$ (Figure 3,b). Moreover, the nano-sized particles were very thin revealed by the presence of fragments. It was clearly seen that stabilizers were adsorbed onto the drug particle surface inhibiting particle growth. The images also revealed that these agglomerates or particle assemblies were composed of a large number of individual nanoparticles.

\section{DSC analysis}

The antisolvent precipitation or HPH process maybe change the physical state of $\mathrm{CXB}$ and, therefore, DSC was performed on the raw CXB, stabilizers, PM and SHSO-CXB. The DSC curve (as Figure 4) of raw CXB showed a single sharp endotherm at $161.49{ }^{\circ} \mathrm{C}$ (with an enthalpy of $-59.33 \mathrm{~J} / \mathrm{g}$ ), which corresponded to its melting point (28). The PM exhibited a slightly broader endotherm than that of raw $\mathrm{CXB}$ at $160.22{ }^{\circ} \mathrm{C}$ attributed to the melting of CXB (with an enthalpy of $-52.32 \mathrm{~J} / \mathrm{g}$ ), which indicated that stabilizers do not change the physical state of $\mathrm{CXB}$ in the physical mixture. However, notable changes involving a broader endothermic peak and a lower enthalpy of $-4.03 \mathrm{~J} / \mathrm{g}$ were observed in SHSO-CXB. Meanwhile, the endotherm at $152.90{ }^{\circ} \mathrm{C}$ was $8.59{ }^{\circ} \mathrm{C}$ lower than that of raw CXB.

\section{X-ray diffraction}

In order to further confirm the physical state of nano-sized CXB, X-ray diffraction was performed. The patterns obtained of raw CXB, $\mathrm{CXB} /$ stabilizer physical mixture and spray-dried powder of $\mathrm{CXB}$ nanosuspensions were displayed in Figure 5. The raw CXB was highly crystallized and exhibited intense crystalline peaks $2 \theta$ values from 15 to $30^{\circ}$, indicating crystalline nature of $\mathrm{CXB}$. The $\mathrm{CXB} /$ stabilizer physical mixture also showed the characteristic crystalline diffraction peaks of CXB (28). However, the characteristic crystalline peaks disappeared in the pattern of SHSO-CXB producing a halo and diffused pattern typical of an amorphous material, revealing that the crystallinity of SHSO-CXB was decreased dramatically. 

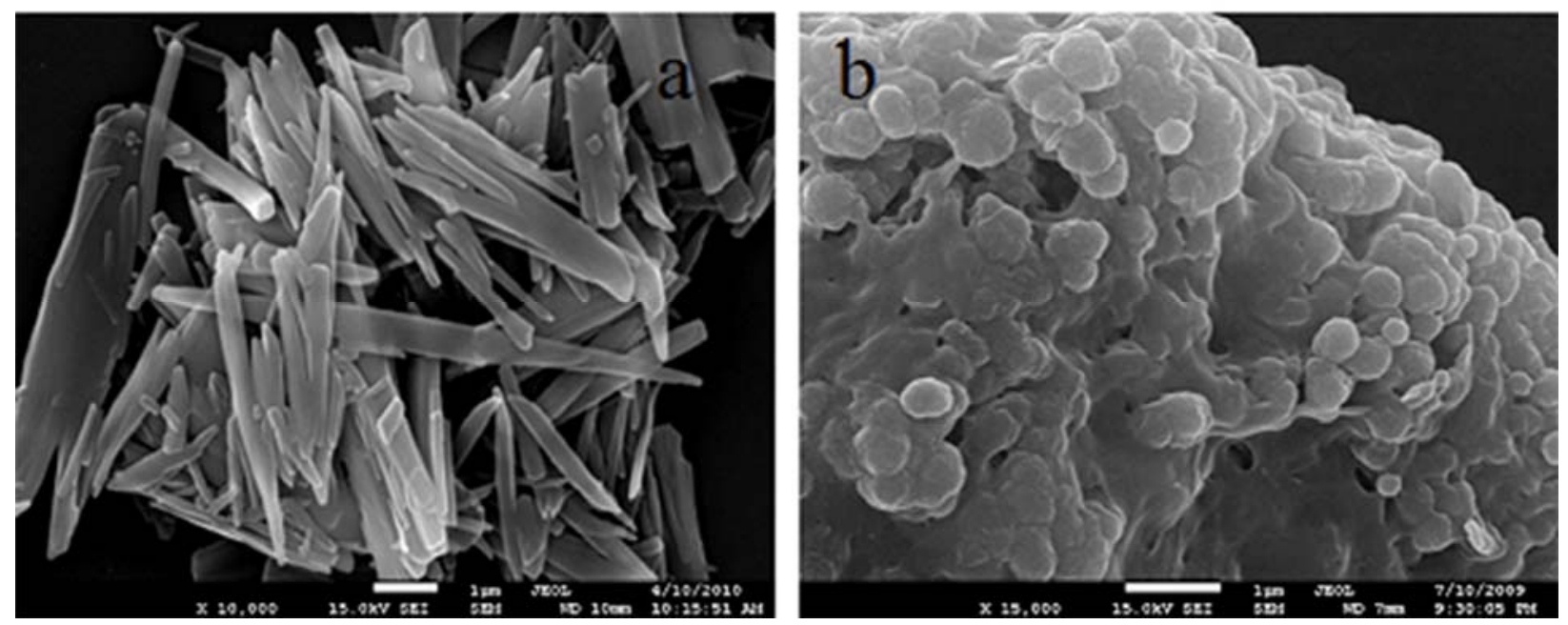

Figure 3. SEM images of raw CXB (a) and CXB nanoparticles (b)

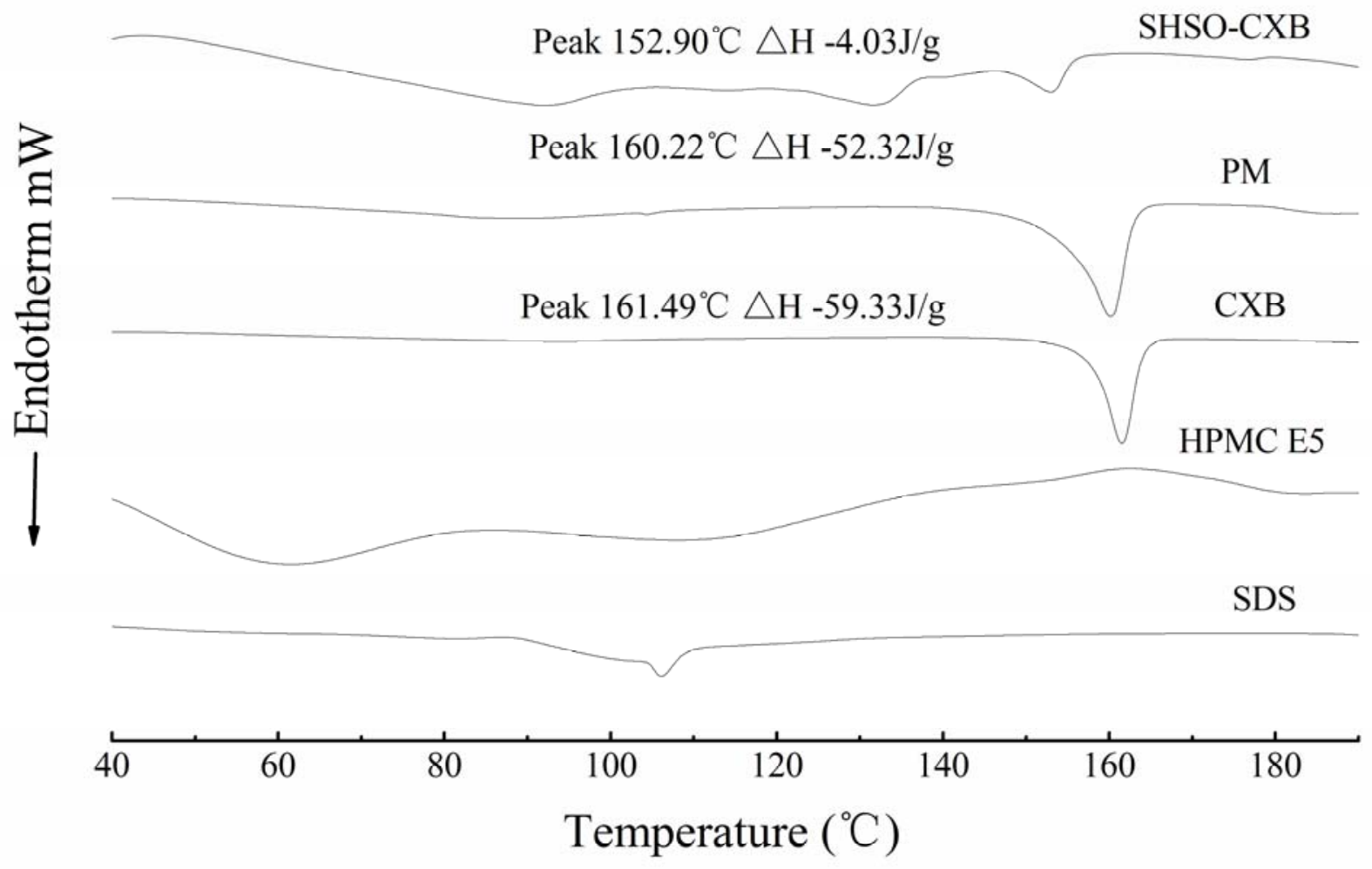

Figure 4. DSC curves: raw CXB; PM; SHSO-CXB and stabilizers (HPMC E5 and SDS) 


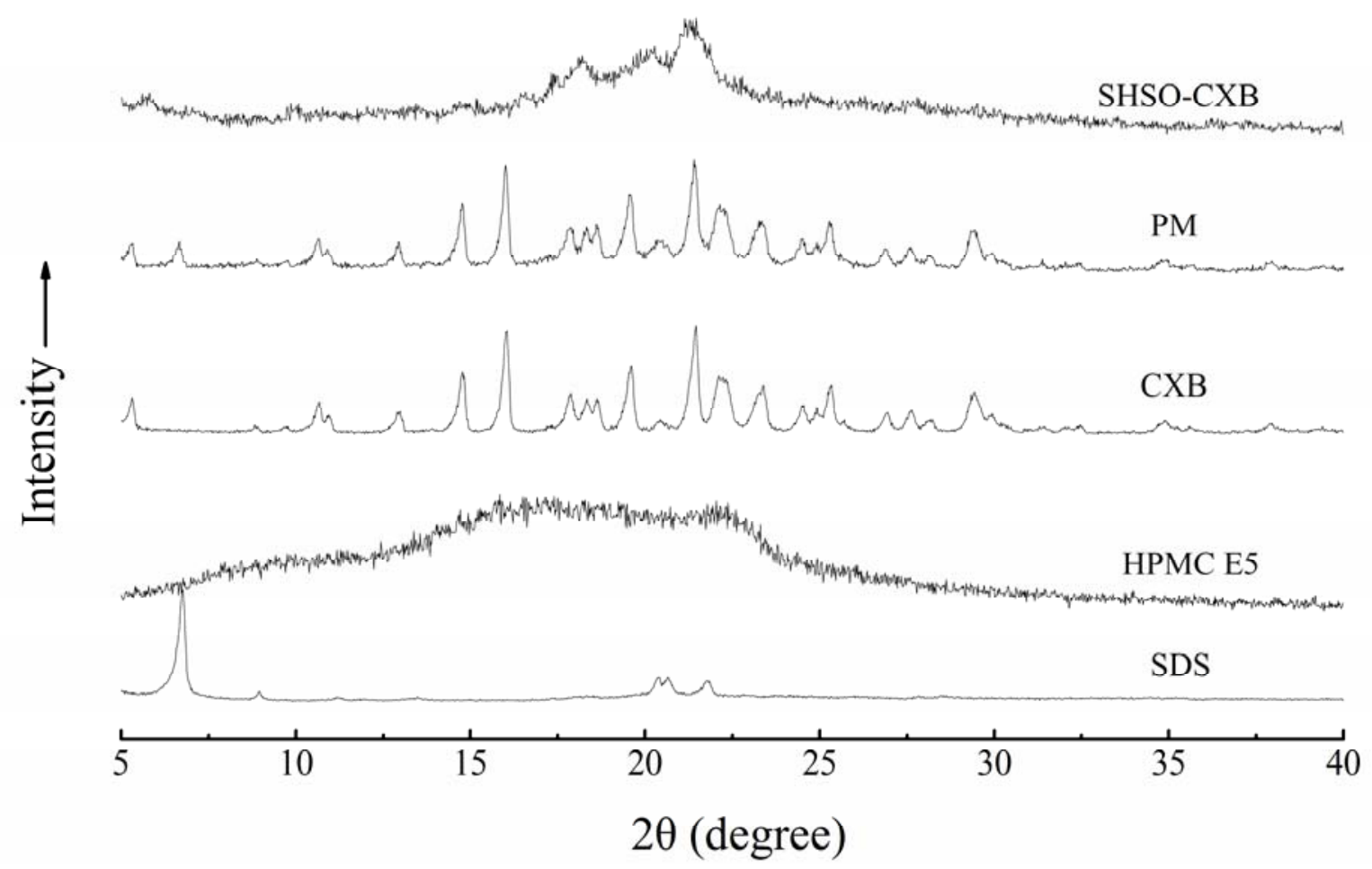

Figure 5. XRPD patterns: raw CXB; PM; SHSO-CXB and stabilizers (HPMC E5 and SDS)

\section{FT-IR spectroscopy}

The interaction between CXB and stabilizers (HPMC and SDS) usually resulted in noticeable changes in the FT-IR spectra. The typical FT-IR spectra of raw $\mathrm{CXB}$, stabilizers and spray-dried powder of CXB nanosuspensions were compared in the range of $4000 \sim 400 \mathrm{~cm}^{-1}$ in Figure 6. It can be seen that the FT-IR spectrum of raw CXB was different from that of SHSO-CXB in the absorption band $3500 \sim 3000 \mathrm{~cm}^{-1}$. The spectrum of CXB displayed characteristic peaks at 3341.3 and $3260.0 \mathrm{~cm}^{-1}$ (sharp doublet for $\mathrm{N}-\mathrm{H}$ stretching), $\quad 1348.5 \mathrm{~cm}^{-1}$ (asymmetric $\mathrm{S}=\mathrm{O}$ stretching) and $1237 \mathrm{~cm}^{-1}$ (C-F stretching). In the case of HPMC E5, a sharp absorption at 3472.3 $\mathrm{cm}^{-1}$ was assigned to the free $\mathrm{O}-\mathrm{H}$ stretching. In the spectrum of SHSO-CXB, the double peaks of $\mathrm{N}-\mathrm{H}$ stretching were much weaker, resulting in a relatively broad absorption band. Meanwhile, the free $\mathrm{O}-\mathrm{H}$ stretching band was observed to shift to a slightly lower wavenumber $\left(3357.7 \mathrm{~cm}^{-1}\right)$. These changes indicated the possibility of hydrogen bonding between the $\mathrm{N}-\mathrm{H}$ group of $\mathrm{CXB}$ and the O-H group of HPMC E5 (29-30). The characteristic peaks in the low frequency region (2000-400 $\left.\mathrm{cm}^{-1}\right)$ were almost unchanged in the spectrum of SHSO-CXB. The close coincidence between the FT-IR spectra of the raw and nano-sized CXB demonstrated that no changes in the CXB chemical structure were caused by preparation processes. In addition, the FT-IR spectrum of PM was identical with the spectrum of raw $\mathrm{CXB}$, indicating that the chemical structure of $\mathrm{CXB}$ was also not influenced by stabilizers in the physical mixture.

\section{$X$-ray photoelectron spectroscopy}

The XPS data for raw CXB and amorphous CXB nanoparticles was summarized in Table 4. It can be clearly seen that the amorphous CXB nanoparticles exhibited a significant difference from the raw $\mathrm{CXB}$ with a relatively higher concentration of $\mathrm{O}$ and lower concentration of $\mathrm{F}$ and $\mathrm{N}$, than the raw CXB surface, indicating a higher presence of hydrophilic $\mathrm{SO}_{2}$ groups. Therefore the intermolecular hydrogen bonding was easily formed between the stabilizer and $\mathrm{CXB}$, and meanwhile stabilizer molecules were also easily adsorbed to the $\mathrm{CXB}$ nanoparticles surface. In addition, a lower presence of $\mathrm{CF}_{3}$ also contributed to the enhancement for wettability of the amorphous CXB nanoparticles due to its high hydrophobic parameter (31). 


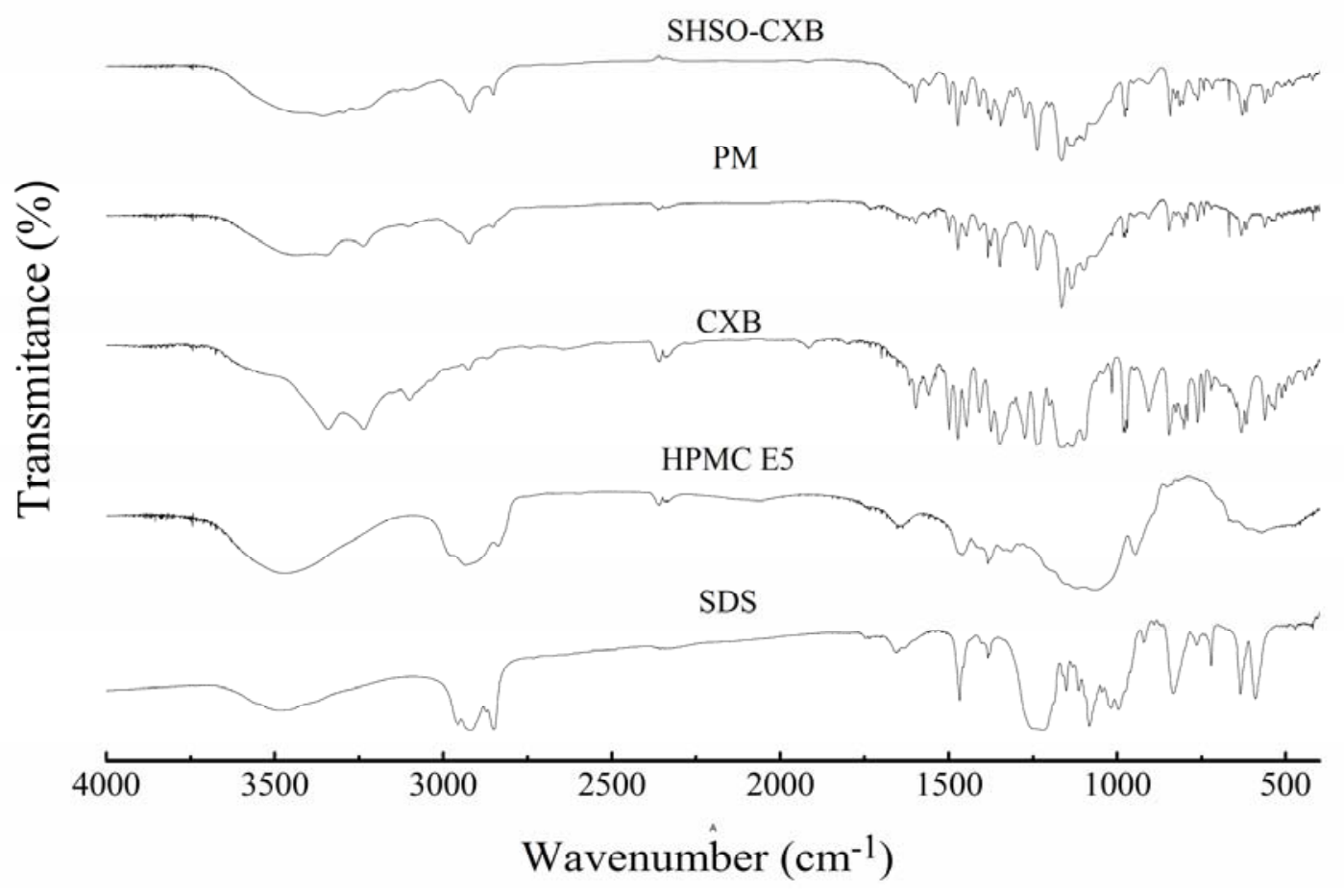

Figure 6. FT-IR spectra: raw CXB; PM; SHSO-CXB and stabilizers (HPMC E5 and SDS)

Table 4. Surface elemental composition of raw CXB and amorphous CXB nanoparticles

\begin{tabular}{lccccc}
\hline \multirow{2}{*}{ Sample } & \multicolumn{5}{c}{ Atomic concentration (\%) } \\
\cline { 2 - 6 } & C 1s & O 1s & N 1s & F 1s & S 2p \\
\hline Amorphous CXB nanoparticles & 65.11 & 14.59 & 7.08 & 8.51 & 4.70 \\
Raw CXB & 66.8 & 8.24 & 9.33 & 11.1 & 4.53 \\
\hline
\end{tabular}

\section{Long term stability}

CXB powder with nanoparticles obtained by spray-drying was kept in a desiccator at room temperature. A disadvantage of the amorphous state is its instability under storage. Hence, the preliminary solid-state stability of the powder with nano-sized CXB was studied for a period of nine months using XRPD as a characterization parameter (peaks arising from the halo pattern) in comparison with the initial sample (Figure 7). It can be seen that there were no obvious characteristic crystalline peaks of CXB appeared in the XRPD pattern and no remarkable increase of intensity of peaks under storage for nine months indicating a good physical stability.

\section{Contact angle analysis}

Wettability is a measure of the ability of a bulk powder to take up a liquid under the influence of capillary forces, and it depends on the particle size, density and porosity of the powder bed. The results of the contact angle were showed in Table 5. The contact angle of raw CXB was $61.59 \pm$ $0.78^{\circ}$ demonstrating high lipophilicity. However, the contact angle of SHSO-CXB was dramatically reduced $\left(16.95 \pm 0.56^{\circ}\right)$ indicating better wettability in comparison with CXB. It also can be seen that the addition of stabilizers (a combination of HPMC E5 and SDS, 2:1, w/w) had an effect on the wettability of CXB due to a reduction in the contact angle of PM. However, the contact angle of SHSO-CXB was smaller than that of PM, further illustrating the better wettability of the spray-dried powder with nano-sized CXB. 


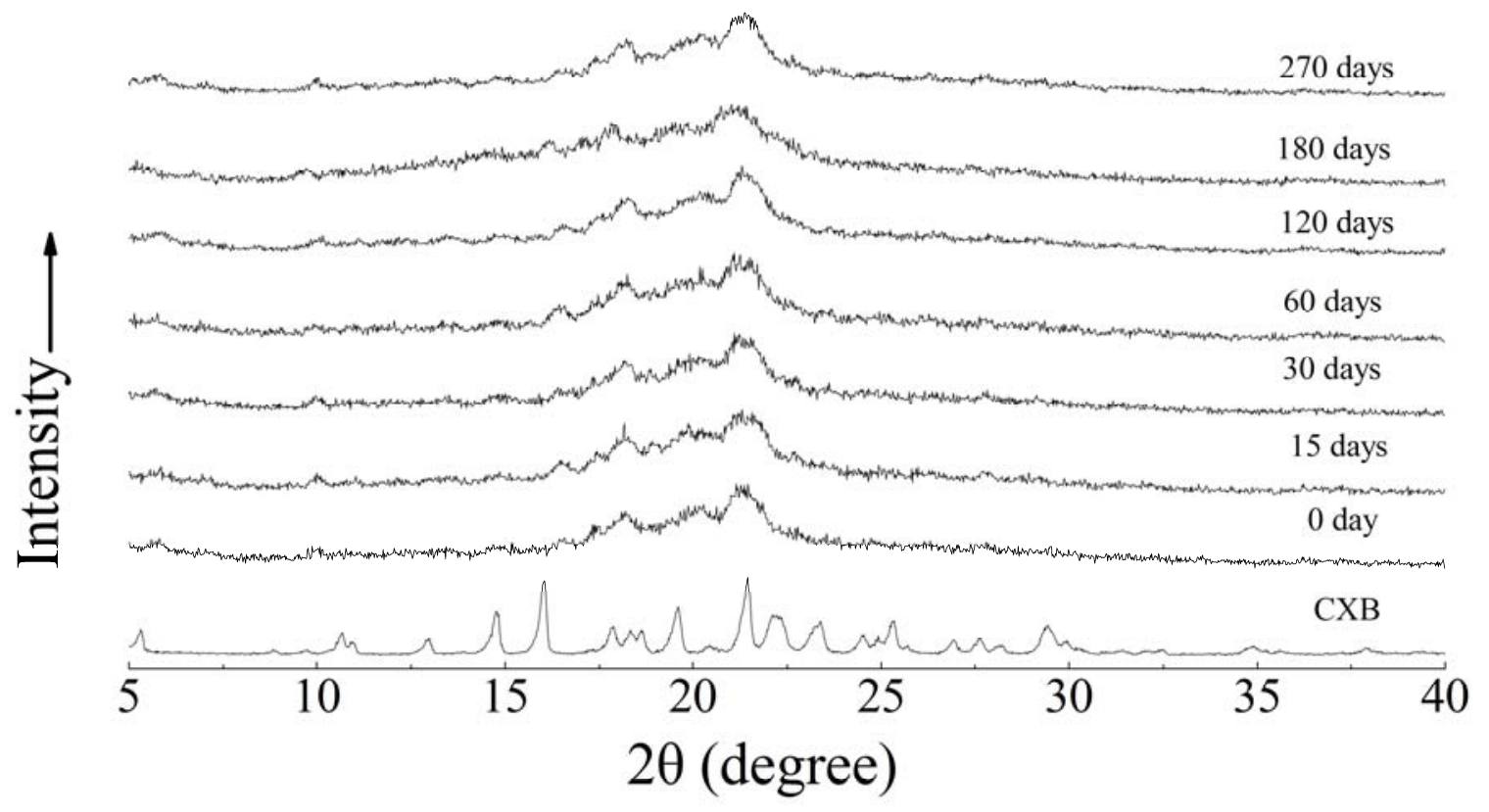

Figure 7. XRPD patterns of SHSO-CXB on different days under storage at room temperature

\begin{tabular}{lc}
\hline Table 5. The contact angles of raw CXB, PM, and SHSO-CXB, Mean \pm S.D., $\mathrm{n}=3$ \\
\hline Sample & Contact angle $\left(^{\circ}\right)$ \\
\hline CXB & $61.59 \pm 0.78$ \\
PM & $27.58 \pm 0.67$ \\
SHSO-CXB & $16.95 \pm 0.56$ \\
\hline
\end{tabular}

\section{Saturation solubility and in vitro dissolution study}

Table 6 showed the saturation solubility of raw $\mathrm{CXB}$ and SHSO-CXB. The results of the solubility study indicated that pure CXB possesses a very low solubility in water $(4.67 \pm 0.78, \mu \mathrm{g} / \mathrm{mL})$ which was consistent with the literature value (17). The saturation solubility of SHSO-CXB $(19.26 \pm 0.63, \mu \mathrm{g} / \mathrm{mL})$ was four times that of raw CXB.

The profiles shown in Figure 8 illustrated the dissolution rates of raw CXB and SHSO-CXB. Nano-sized CXB displayed a dramatic increase in the rate and extent of dissolution in comparison with raw $\mathrm{CXB}$, especially during the initial stage (first $5 \mathrm{~min}$ ). SHSO-CXB exhibited $72 \%$ drug dissolution within $1 \mathrm{~min}$ whereas only $17 \%$ of raw CXB dissolved during the same period. After 5 min, SHSO-CXB was dissolved completely, but only $30 \%$ of raw CXB had dissolved, owing to its crystalline nature and larger crystal size. The dissolution profiles of PM was similar to that of raw $\mathrm{CXB}$, which showed that the mechanical physical mixing of raw $\mathrm{CXB}$ and stabilizers had little effect on the dissolution of raw CXB.

\section{Bioavailability}

The in-vivo study in beagle dogs was performed to quantify concentration, after administering formulations of SHSO-CXB orally in suspension form. The plasma concentration in beagle dogs following oral administration of the Celecoxib Capsules and SHSO-CXB were compared. A summary of pharmacokinetic parameters and plasma concentration versus time curves was showed in Table 7 and Figure 9, respectively. The plasma concentration profile for SHSO-CXB represented significant improvement in drug absorption compared with the Celecoxib Capsules. The $\mathrm{C}_{\max }$ and $\mathrm{AUC}_{0-24 \mathrm{~h}}$ values of SHSO-CXB were approximately three fold, two fold greater than those of the Celecoxib Capsules respectively, indicating a remarkable improvement in the oral absorption of SHSO-CXB when administered in the form of amorphous nanoparticles. 
Table 6. The saturated solubility: raw CXB; PM; SHSO-CXB, Mean \pm S.D., $n=3$

\begin{tabular}{lc}
\hline Sample & Saturated solubility $(\mu \mathrm{g} / \mathrm{mL})$ \\
\hline CXB & $4.67 \pm 0.78$ \\
PM & $5.28 \pm 0.61$ \\
SHSO-CXB & $19.26 \pm 0.63$ \\
\hline
\end{tabular}

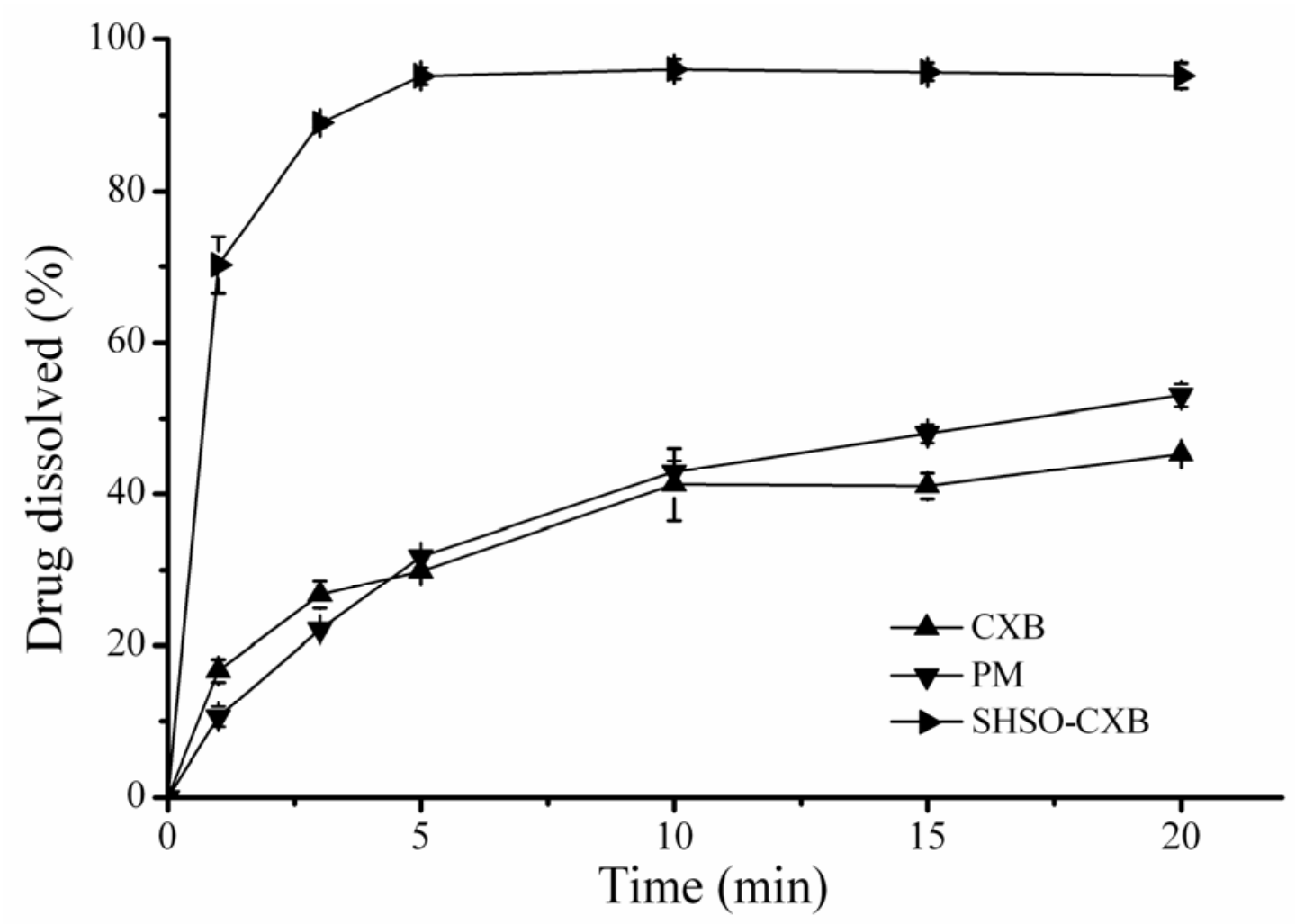

Figure 8. The dissolution profiles: raw CXB; PM; SHSO-CXB, $n=3$

Table 7. Pharmacokinetic parameters of Celecoxib Capsules and SHSO-CXB after oral dose in beagle dog, Mean \pm S.D., $\mathrm{n}=6$

\begin{tabular}{lcc}
\hline Pharmacokinetic parameter & Celecoxib Capsules & SHSO-CXB \\
\hline $\mathrm{C}_{\max }(\mathrm{ng} / \mathrm{mL})$ & $315.9 \pm 25.8^{*}$ & $923.9 \pm 31.0^{*}$ \\
$\mathrm{~T}_{\max }(\mathrm{h})$ & 3.0 & 1.5 \\
$\mathrm{AUC}$ & $2575.3 \pm 21.2^{*} * 4 \mathrm{~h}$ & $(\mathrm{ng} \cdot \mathrm{h} / \mathrm{mL})$ \\
$* \mathrm{P}<0.05$ & & $4807.0 \pm 34.3^{*}$ \\
\hline
\end{tabular}




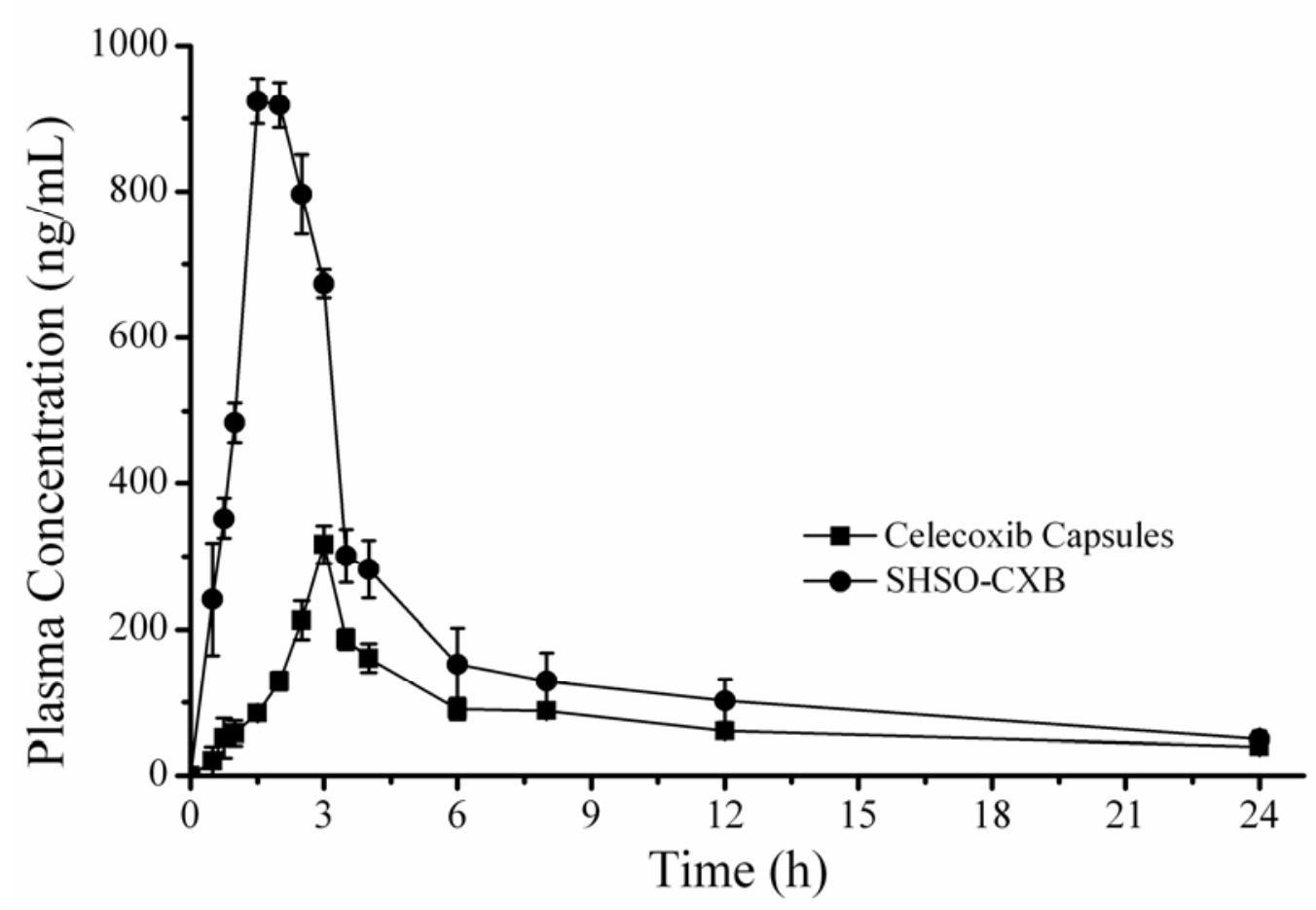

Figure 9. Representative plasma concentration versus time profiles after oral administration of Celecoxib Capsules and SHSO-CXB in beagle dog, $\mathrm{n}=6$

\section{DISCUSSION}

\section{Preparation, particle size and morphology of CXB nanoparicles}

The CXB nanoparticles were produced by the following two steps. Initially, antisolvent precipitation experiments were carried out with acetone as solvent due to its high capacity to solubilize CXB and with water as the antisolvent under sonication at $4{ }^{\circ} \mathrm{C}$. Preliminary sonoprecipitation experiments were carried out by varying the sonication amplitude, sonication time and processing temperature. Precipitation at 300 $\mathrm{W}$ for intermittent sonication of $3 \mathrm{~s}$ at every $2 \mathrm{~s}$ intervals for a total sonication time of $3 \mathrm{~min}$ was found to be the most suitable. Processing at a lower temperature resulted in rapid precipitation with smaller particles. Hence, all the experiments were carried out at $4{ }^{\circ} \mathrm{C}$ and $300 \mathrm{~W}$ for $3 \mathrm{~min}$. As can be seen from Table 3, smaller particle size and narrower PSD of CXB nanoparticles than that prepared only by sonication was obtained by the combination of antisolvent precipitation under sonication and HPH. Although some advantages of sonication favor the formation of nanoparticles, our studies suggested that it was difficult to produce CXB nanoparticles with a narrow PSD only by antisolvent precipitation under sonication due to many process parameters. Actually, the reproducibility of particle size of CXB nanoparticles under sonication was poor. Therefore the CXB suspensions was further homogenized after sonication. It is evident that the uniformity of CXB particle size is significantly improved after homogenization (Table 3, Figure 2, 500 bar), which should be more beneficial to enhance the stability of CXB nanoparticles. The spray-dried powder was re-dispersed and its particle size was consistent with the initial size (Table 3), indicating that the particle size of CXB nanoparticles was not affected by spray-drying. Additionally, the nanosuspensions were stored in a refrigerator $\left(4{ }^{\circ} \mathrm{C}\right)$ for two months without obvious increase in particle size, which suggested that stability of CXB nanoparticles was also not bad. The above results demonstrated that the combination of antisolvent precipitation under sonication and $\mathrm{HPH}$ offered a better and more promising method of producing $\mathrm{CXB}$ nanoparticles.

A combination of HPMC E5 and SDS (2:1, $\mathrm{w} / \mathrm{w}$ ) was the most successful of all the stabilizing agents investigated as far as the formation of CXB suspensions were concerned. The results indicated that HPMC E5 and SDS played a key role in inhibiting the growth of $\mathrm{CXB}$ 
nanoparticles. This phenomenon can be explained by a steric barrier and electrostatic repulsion. HPMC is a polymer containing a number of methoxyl and hydroxypropyl groups, and the hydrophobic parts have good affinity for drug particles and can be adsorbed onto the drug particle surface providing an effective steric barrier against growth. In addition, hydrogen bonds can form between the drug molecule and HPMC. SDS is an anionic surfactant, which provides effective electrostatic repulsion between CXB nanoparticles to inhibit particles aggregation. Many reports have shown that if preliminary particles can be arrested efficiently by appropriate stabilizers, the nanosuspensions system can be maintained for a longer time (32). In this method, uniform and small CXB particles can be produced at low homogenization pressure (Figure 2, 500 bar). It indicated that a very high pressure and a large number of cycles were not needed, so the drug damage caused by the high energy generated during the HPH process can be reduced greatly. Actually, the temperature will increase dramatically when homogenization pressure exceed 600 bar even though the cooling water was always cycled during production. The low temperature at low homogenization pressure should be favorable to keep the properties of active drugs, especially for the heat sensitive active pharmaceutical ingredients.

In Figure 3, the CXB nanoparticles was spherical as compared with raw CXB. On one hand, the stabilizers make the nanoparticles stable. On the other hand, they change the crystal habit of $\mathrm{CXB}$, resulting in the formation of the spherical particles. Generally, CXB exists as long needle-shaped crystals that have undesirable properties such as cohesiveness, low bulk density and compressibility. They therefore tend to separate out and agglomerate leading to poor blend uniformity and formation of a monolithic mass upon compression in the tablet die, making successful tabletting very problematical $(24,28)$. However, the spherical particles were expected to improve the powder properties of CXB, such as flowability and compressibility. Venkadari Rammohan Gupta et al. (33) have obtained the spherical CXB crystals exhibited satisfactory micromeritic properties. A.R. Paradkar et al. (34) have obtained spherical crystallization of CXB by the solvent change method exhibited decreased crystallinity and satisfactory micromeritic, mechanical, and compressional properties.

\section{Physicochemical properties and mechanism of dissolution enhancement}

The low shift of the melting peak and the considerable reduction of the corresponding enthalpy (Figure 4) were caused by a reduction in the particle size of CXB and a remarkable decrease of the degree of crystallinity that can be confirmed by XRPD pattern of SHSO-CXB (Figure 5), but this also indicated that $\mathrm{CXB}$ and the stabilizers (a combination of HPMC E5 and SDS, $2: 1, w / w)$ might have interacted. During the preparation of SHSO-CXB, three different processes- antisolvent precipitation under sonication, HPH and spray-drying were involved. According to many reports, they all have the possibilities that decrease the degree of crystallinity of drug compounds or transform the drug crystals to its amorphous form (8). Therefore it is significant to investigate the effect of the above three processes on the physical state of $\mathrm{CXB}$. The investigations were performed by obtaining the solid powder of CXB produced by different processes and the solid powder were characterized by XRPD. The XRPD patterns of CXB powder obtained in every preparation process (antisolvent precipitation under sonication, HPH and spray-drying) were showed in Figure 10. It can be clearly seen that several characteristic crystalline peaks at $10-15^{\circ}$ and $22-30^{\circ}$ disappeared and other characteristic crystalline peaks at $15-22^{\circ}$ broadened obviously in the pattern of FVF-CXB. However, other two XRPD patterns of SSO-CXB and SHSO-CXB were nearly the same as that of FVF-CXB. The results indicated that the process of antisolvent precipitation under sonication resulted in the dramatic decrease of the crystallinity of CXB instead of spray-drying and HPH. The rapid nucleation of $\mathrm{CXB}$ in antisolvent precipitation under sonication and the effects of stabilizer possibly led to a random arrangement of the $\mathrm{CXB}$ molecules resulting in amorphous $\mathrm{CXB}$ nanoparticles. This phenomenon can be explained by the fact that under fast nucleation rate, the drug solute lacks sufficient time to incorporate into the growing crystal lattice accurately to form perfect crystals (6) and suppression of stabilizer. Ravindra S. Dhumal et al. (11) have prepared amorphous cefuroxime axetil nanoparticles by sonoprecipitation. It has been known that a change in the physical state of a drug from a crystalline to an amorphous form leads to a high energy state and high disorder, contributing to an increase in the dissolution rate and oral bioavailability. 


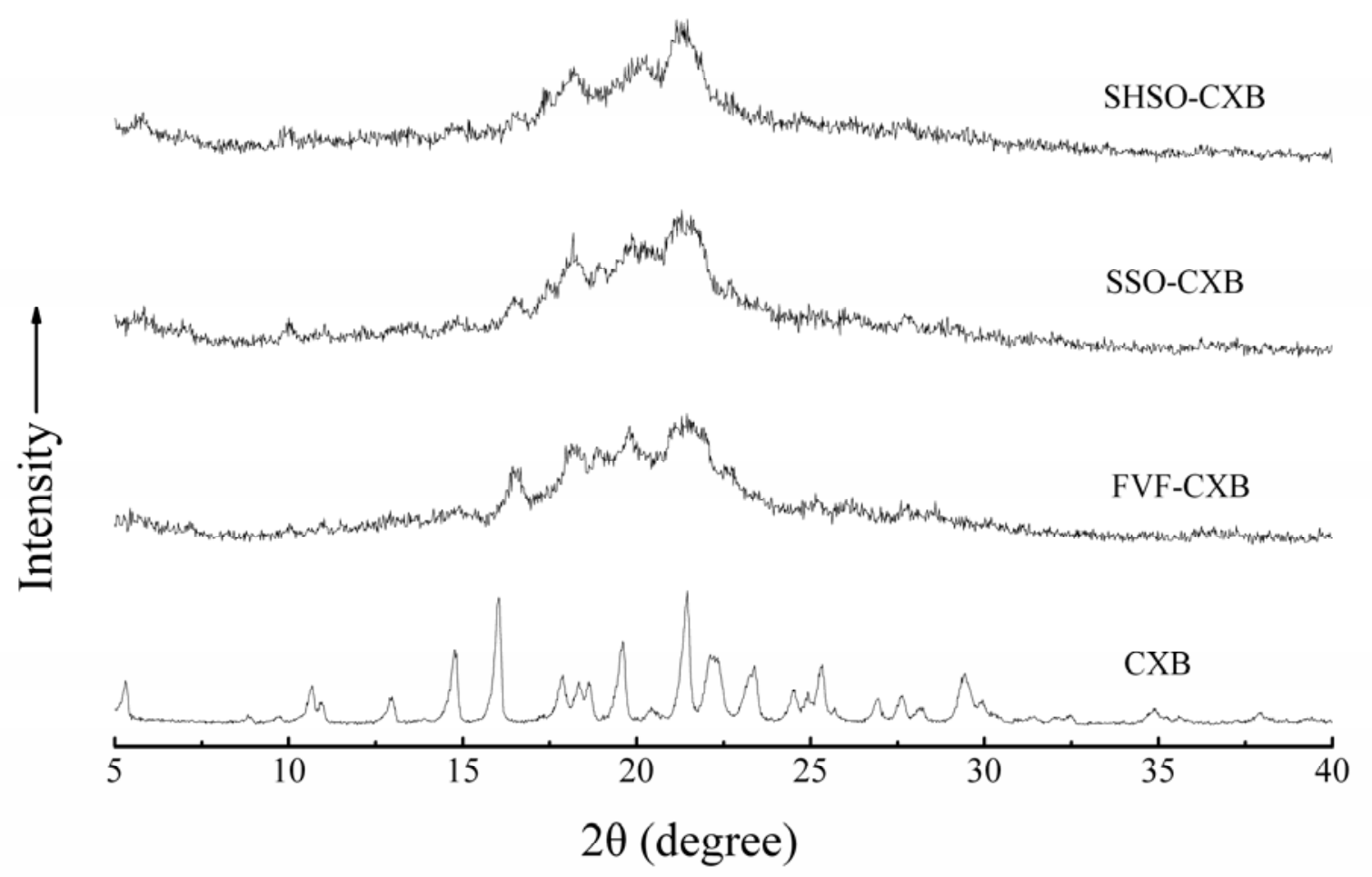

Figure 10. XRPD patterns: raw CXB; FVF-CXB, powder solidified by flocculation and vacuum filtration of CXB suspensions prepared only by antisolvent precipitation under sonication; SSO-CXB, spray-dried powder of CXB suspensions prepared only by antisolvent precipitation under sonication; SHSO-CXB, spray-dried powder of CXB nanosuspensions prepared by the combination of antisolvent precipitation under sonication and HPH.

It is crucial of the selection of stabilizers during preparing the nano-sized particles. The drug nanoparticles was easy to form and more stable especially in liquid system if interactions existed between the drug compounds and stabilizers, which was because of the reason that the particles growth and agglomerate can be arrested effectively by stabilizers. The formation of hydrogen bonding was the most common among the interactions between the active substances and excipients. The intermolecular hydrogen bonding between CXB and polymers (as discussed in results) was beneficial to the formation of CXB nanoparicles and its stability. Actually, it is evident from the results of the long term stability of nano-sized CXB (Figure 7). In Figure 7, it can be seen that there were no obvious characteristic crystalline peaks of CXB appeared in the XRPD pattern under storage for nine months indicating a good physical stability, which probably because the reduction of particle size of $\mathrm{CXB}$ and interactions between CXB and stabilizer prevent $\mathrm{CXB}$ crystallization. Additionally, the intermolecular hydrogen bonding between CXB and polymers was contributed to the improved wettability of the particles, saturation solubility and dissolution rate.

$\mathrm{X}$-ray photoelectron spectroscopy was used to investigate the surface chemistry of raw $\mathrm{CXB}$ and amorphous CXB nanoparticles, because surface chemistry of drugs governs surface polarity and the interactions with the external environment. In the $\mathrm{CXB}$ molecule (Figure 1), $\mathrm{O}$ atoms of the sulphonyl group $\left(\mathrm{SO}_{2}\right), \mathrm{N}$ of the pyrazole ring and $\mathrm{F}$ of the trifluoromethyl $\left(\mathrm{CF}_{3}\right)$ group are electron donating centers, and the amido hydrogens are electron accepting centers (35-36). These groups would have propensity to form hydrogen bond with neighbouring like/unlike molecules and thus contribute to the hydrophilic/polar sites on the molecule. As a result, Intermolecular hydrogen bonding was easily formed between the stabilizer and $\mathrm{CXB}$, and meanwhile stabilizer molecules were also easily adsorbed to the CXB nanoparticles surface due to a higher presence of hydrophilic $\mathrm{SO}_{2}$ groups and a lower presence of $\mathrm{CF}_{3}$ in the surface of the amorphous CXB nanoparticles than those of raw CXB (Table 4). 
Similarly, the results of XPS data played a very important role in the formation and stability of CXB nanoparticles. But even more important, the wettability of CXB would increase significantly owing to a higher presence of hydrophilic $\mathrm{SO}_{2}$ groups and a lower presence of hydrophobic $\mathrm{CF}_{3}$ in virtue of its high hydrophobic parameter (log octanol- water partition coefficient $=0.88$ ) (31) would also impart lipophilicity to the molecule. The XPS data was correlated with the molecular structural arrangements due to different molecular structural arrangements of raw $\mathrm{CXB}$ and amorphous CXB nanoparticles (35-36). For the increased wettability, it is evident from the dramatic decrease of contact angle of nano-sized CXB compared with that of raw CXB (Table 5). The improved wettability of the powder with nano-sized CXB was contributed to the increase in the saturation solubility and dissolution rate.

\section{Saturation solubility, in vitro dissolution and in vivo bioavailability}

In the present work, the aqueous solubility of CXB was improved greatly. The saturation solubility of nano-sized CXB $(19.26 \mu \mathrm{g} / \mathrm{mL})$ was four times greater than that of raw CXB (4.67 $\mu \mathrm{g} / \mathrm{mL}$ ). The reduction of the particle size of CXB to nano scale, increased surface area and enhanced hydrophility were responsible for the significantly increased saturation solubility. In addition, the in vitro dissolution of nano-sized $\mathrm{CXB}$ was excellent in comparison with that of raw CXB (Figure 8). In Figure 8, SHSO-CXB exhibited $72 \%$ drug dissolution within $1 \mathrm{~min}$ whereas only $17 \%$ of raw CXB dissolved during the same period. After $5 \mathrm{~min}$, SHSO-CXB was dissolved completely, but only $30 \%$ of raw CXB had dissolved, owing to its crystalline nature and larger crystal size. According to Noyes-Whitney equation, the solid dissolution rate is directly proportional to its surface area exposed to the dissolution medium. The increased dissolution rate of SHSO-CXB could be attributed to the combination of effects like amorphization, better wettability of the surface of the drug particles and particle size which was reduced to nano scale, greatly increasing the specific surface area and decreasing diffusion layer thickness. These promising results in in-vitro drug release studies promoted us to perform in-vivo studies in dogs.

The in vivo study of nano-sized CXB and Celecoxib Capsules in beagle dogs was performed in order to evaluate the bioavailability of CXB nanoparticles. It is evident that nano-sized CXB was rapidly absorbed and reached maximum concentrations by $1.5 \mathrm{~h}$ in dogs as compared with Celecoxib Capsules (Figure 9). Additionally, the
$\mathrm{C}_{\max }$ and $\mathrm{AUC}_{0-24 \mathrm{~h}}$ values of SHSO-CXB were approximately three fold, two fold greater than those of the Celecoxib Capsules respectively, indicating a remarkable improvement in the oral absorption of SHSO-CXB when administered in the form of suspensions (Table 7). SHSO-CXB was found to exhibit better bioavailability as compared to Celecoxib Capsules because of its greater dissolution rate owing to its amorphization, increased wettability and reduced particle size with increased surface area and reduced diffusion layer thickness. This was in agreement with the in-vitro dissolution studies. The absolute bioavailability of $\mathrm{CXB}$ in polyethylene glycol/saline after oral administration in beagle dogs was $64-88 \%$ and $22-40 \%$ when given in capsules. CXB given to humans in capsules with a high fat meal exhibited only a slight increase $(11 \%)$, but that is not clinically significant with regard to safety or efficacy. Moreover, inter-individual variability of $\mathrm{CXB}$ plasma profiles in humans was also signally high. CXB is classified as a BSC class II substance which suggest that it is a highly permeable drug that can be absorbed throughout the gastrointestinal tract and that dissolution is a rate limiting factor for absorption from solid dosage forms (18). Hence, increasing the aqueous solubility and dissolution rate of CXB is expected to enhance its overall oral bioavailability. In this present study, the SHSO-CXB exhibited significantly increased saturation solubility and dissolution rate. Additionally, the nano-sized CXB exhibited enhanced bioavailability in comparision with Celecoxib Capsules in dogs. Therefore, the absolute bioavailability of nano-sized CXB in humans should be high according to the above analysis. Certainly, the deduction needs to be further validated by some experiments.

CXB concentration in common commercial products varies between 100 and $200 \mathrm{mg}$. The enhanced drug dissolution and bioavailability of nano-sized CXB suggest that the dose of CXB can be reduced when used as a dosage form from either alone or in the form of other solid delivery systems such as capsule and tablet forms. The reduction of drug dose is not only favorable economically but also is desirable in decreasing its side effects especially when administered in multiple dosage regiments.

\section{CONCLUSION}

The stable amorphous CXB nanoparticles were successfully prepared by antisolvent precipitation under sonication followed by HPH and the nanosuspensions were converted into dry powders 
by spray-drying. The amorphous CXB in nanoparticles formed in the procedure of antisolvent precipitation under sonication and kept the state during HPH and spray-drying. The amorphous CXB nanoparticles exhibited different surface property and improved wettability compared with the CXB crystal due to different molecular structural arrangements. The amorphous CXB nanoparticles showed dramatic improvement in rate as well as extent of in-vitro drug dissolution and oral bioavailability in beagle dogs. The improvement can be attributed to amorphization, better wettability, increased saturation solubility and surface area, reduced particle size and decreased diffusion layer thickness.

\section{ACKNOWLEDGEMENTS}

This work was supported by the National Basic Research Program of China (973 Program) (No. 2009CB930300) and Major National Platform for Innovative Pharmaceuticals (No. 2009ZX09301-021). In addition, we gratefully acknowledge Dr. David Jack for checking and editing the manuscript, who is a native English-speaker with sufficient scientific knowledge.

\section{REFERENCES}

1. Radtke M. Pure drug nanaoparticles for the formulation of pooly soluble drugs. New Drugs, 2001; 3: 62-68.

2. Lipinski C. Poor aqueous solubility-an industry wide problem in drug discovery. Am Pharm Rev, 2002; 5: 82-85.

3. Dressmann JB, Reppas C. In vitro-in vivo correlations for lipophilic, poorly water-soluble drugs. Eur J Pharm Sci, 2000; 11: 73-80.

4. Kesisoglou F, Panmai S, Wu Y. Nanosizing-oral formulation development and biopharmaceutical evaluation. Adv Drug Deliv Rev, 2007; 5916: 31-44.

5. Patravale VB, Date AA, Kulkarni RM. Nanosuspensions: a promising drug delivery strategy. J Pharm Pharmacol, 2004; 56: 827-840.

6. Rabinow BE. Nanosuspensions in drug delivery. Nature rev Drug Disc, 2004; 3: 785-796.

7. Wong S M, Kellaway IW, Murdan S. Enhancement of the dissolution rate and oral absorption of a poorly water soluble drug by formation of surfactant-containing microparticles. Int J Pharm, 2006; 317: 61-68.

8. Muller RH, Jacobs C, Kayser O. Nanosuspension as particulate drug formulations in therapy rationale for development and what we can expect for the future. Adv Drug Deliv Rev, 2001; 47: 3-19.

9. Guo Z, Zhang M, Li H, Wang E, Kougouslos E. Effect of ultrasound on antisolvent crystallization process. J Cryst Growth, 2005; 273: 555-563.

10. Louhi-Kultanen M, Karjalainen M, Rananen J, Huhtanen M, Kallas J. Crystallization of glycine with ultrasound. Int J Pharm, 2006; 320: 23-29.

11. Dhumal Ravindra S, Biradar Shailesh V, Yamamura Shigeo, Paradkar Anant R, York Peter. Preparation of amorphous cefuroxime axetil nanoparticles by sonoprecipitation for enhancement of bioavailability. Eur J Pharm Biopharm; 2008; 70: 109-115.

12. Verma Sudhir, Gokhaleb Rajeev, Burgessa Diane J. A comparative study of top-down and bottom-up approaches for the preparation of micro/nanosuspensions. Int J Pharm, 2009; 380: 216-222.

13. Amidon GL, Lennernas H, Shah VP, Crison JR. A theoretical basis for a biopharmaceutic drug classification: the correlation of in vitro drug product dissolution and in vivo bioavailability. Pharm Res, 1995; 12: 413-420.

14. Detrembleur C, De Nayer J, Den Hecke A Van. Celecoxib improves the efficiency of the locomotor mechanism in patients with knee osteoarthritis. A randomised, placebo, double-blind and cross-over trial. Osteoarthr Cartilage, 2005; 13: 206-210.

15. Kivitz AJ, Espinoza LR, Sherrer YR, Liu-Dumaw $\mathrm{M}$, West CR. A comparison of the efficacy and safety of celecoxib $200 \mathrm{mg}$ and celecoxib $400 \mathrm{mg}$ once daily in treating the signs and symptoms of psoriatic arthritis. Semin Arthritis Rheum, 2007; 37: 164-173.

16. Yelland MJ, Nikles CJ, Mcnairn N, Del Mar CB, Schluter PJ, Brown RM. Celecoxib compared with sustained-release paracetamol for osteoarthritis: a series of n-of-1 trials. Rheumatology (Oxford), 2007; 46: 135-140.

17. Reddy MN, Rehana Tasneem, Ramakrishna S, Chowdary KPR, Diwan PV. $\beta$-cyclodextrin complexes of celecoxib: molecular-modeling characterization, and dissolution studies. AAPS PharmSci,2004; 6 (1): 1-9.

18. Paulson SK, Vaughn MB, Jessen SM, Lawal Y, Gresk CJ, Yan B, Maziasz TJ, Cook CS, Karim A. Pharmacokinetics of celecoxib after oral administration in dogs and humans: Effect of food and site of absorption. J Pharmacol Exp Ther, 2001; 297: 638-645.

19. Shono Y, Jantratid E, Janssen N, Kesisoglou F, Mao Y, Vertzoni M, Reppas C, Dressman JB. Prediction of food effects on the absorption of celecoxib based on biorelevant dissolution testing coupled with physiologically based 
pharmacokinetic modeling. Eur J Pharm Biopharm, 2009; 73: 107-114.

20. Rawat S, Jain SK. Solubility enhancement of celecoxib using $\beta$-cyclodextrin inclusion complexes. Eur J Pharm Biopharm, 2004; 57: 263-267.

21. Subramanian N, Ray S, Ghosal SK, Bhadra R, Moulik SP. Formulation design of self-microemulsifying drug delivery systems for improved oral bioavailability of celecoxib. Biol Pharm Bull, 2004; 27: 1993-1999.

22. Garti N, Avrahami M, Aserin A. Improved solubilization of celecoxib in utype nonionic microemulsions and their structural transitions with progressive aqueous dilution. J Colloid Interface Sci, 2006; 299: 352-365.

23. Homar M, Ubrich N, El Ghazouani F, Kristl J, Kerc J, Maincent P. Influence of polymers on the bioavailability of microencapsulated celecoxib. J Microencapsul, 2007; 24: 621-633.

24. Andrej Dolenc, Julijana Kristl, Saša Baumgartner, Odon Planinšek. Advantages of celecoxib nanosuspension formulation and transformation into tablets. Int J Pharm, 2009; 376: 204-212.

25. Patlolla Ram R, Chougule Mahavir, Patel Apurva R, Jackson Tanise, Tata Prasad NV, Singh Mandip. Formulation, characterization and pulmonary deposition of nebulized celecoxib encapsulated nanostructured lipid carriers. J Control Release, 2010; 144: 233-241.

26. Margulis-Goshen Katrin, Kesselman Ellina, Danino Dganit, Magdassi Shlomo. Formation of celecoxib nanoparticles from volatile microemulsions. Int J Pharm, 2010; 393: 230-237.

27. Bunjes Heike, Unruh Tobias. Characterization of lipid nanoparticles by differential scanning calorimetry, X-ray and neutron scattering. Adv Drug Deliv Rev, 2007; 59: 379-402.

28. Chawla G, Gupta P, Thilagavathi R, Chakraborti A, Bansal AK. Characterization of solid-state forms of celecoxib. Eur J Pharm Sci, 2003; 20: 305-317.

29. Raghavan SL, Schuessel K, Davis A, Hadgraft J. Formation and stabilisation of triclosan colloidal suspensions using supersaturated systems. Int $\mathrm{J}$ Pharm, 2003; 261: 153-158.

30. Li XS, Wang JX, Shen ZG, Zhang PY, Chen JF, Yun J. Preparation of uniform prednisolone microcrystals by a controlled microprecipitation method. Int J Pharm, 2007; 342, 26-32.

31. Smart BE. Fluorine substituent effect. J Fluorine Chem, 2001; 109: 3-11.

32. Dong Yuancai, Ng Wai Kiong, Shen Shoucang, Kim Sanggu, Tan Reginald BH. Preparation and characterization of spionolactone nanoparticles by antisolvent precipitation. Int J Pharm, 2009; 375: 84-88.

33. Gupta Venkadari Rammohan, Mutalik Srinivas, Patel Madhobhai M, Jani Girsh K. Spherical crystals of celecoxib to improve solubility, dissolution rate and miromeritic properties. Acta Pharm, 2007; 57: 173-184.

34. Paradkar AR, Pawar AP, Chordiya JK, Patil VB, Ketkar AR. Spherical crystallization of celecoxib. Drug Dev Ind Pharm, 2002; Vol. 28, No. 10: pp. 1213-1220.

35. Gupta P, Bansal AK. Devitrification of amorphous celecoxib. AAPS PharmSciTech, 2005; 6: E223-E230.

36. Kaushal AM, Bansal AK. Thermodynamic behavior of glassy state of structurally related compounds. Eur J Pharm BioPharm, 2008; 69: 1067-1076. 